\title{
Phase Noise Robust Optical Heterodyne System for Reduced Complexity Millimeter-Wave Analog Radio-over-Fibre

\author{
Colm Browning ${ }^{*}$, Amol Delmade ${ }^{1}$, Yi Lin ${ }^{1}$, Julien Poette ${ }^{2}$, \\ Hamza Hallak Elwan ${ }^{2}$ and Liam P. Barry ${ }^{1}$
}

\author{
${ }^{1}$ School Of Electronic Engineering, Dublin City University, Glasnevein, Dublin D09W6Y4, Ireland \\ ${ }^{2}$ Institute of Microelectronics, Electromagnetism and Photonics, Grenoble Alpes University, Grenoble, France \\ *colm.browning@dcu.ie \\ Keywords: MILLIMETER-WAVE, X-HAUL, ANALOG RADIO-OVER-FIBER, OPTICAL HETERODYNE
}

\section{Abstract}

Strict optical linewidth/coherence requirements for A-RoF systems are overcome through development of an analog optical heterodyne architecture tolerant to phase noise and carrier offset. Successful generation and reception of a 60GHz UF-OFDM signal using two free-running tunable lasers, without digital phase/frequency offset compensation, is demonstrated.

\section{Introduction}

As demand for bandwidth continues to intensify with the advent of new applications and the dawn of the $5 \mathrm{G}$ era, new technologies which facilitate the distribution and dense deployment of high speed wireless transceivers are of critical importance. The use of millimeter-wave (mm-wave) frequencies for the wireless provision of bandwidth intensive applications such as Virtual Reality (VR), live Ultra High Definition (UHD) video streaming and autonomous driving is considered to be a key enabling technology for 5G communications and beyond [1]. 5G New Radio (NR) has already identified mm-wave bands in the 25 and $40 \mathrm{GHz}$ ranges for wireless transmission, and bands around 60 and 90 $\mathrm{GHz}$ are also under intense examination with many successful filed trials reported for each [2-4].

Considering the difficulty and expense involved with distributing and generating high quality $\mathrm{mm}$-wave carriers purely by electronic means, optical techniques, such as optical heterodyning, which are inherently compatible with Radioover-Fiber (RoF) front/cross/back-haul (x-haul), have been the subject of much research in this vein $[5,6]$. Currently deployed, Digital RoF (D-RoF) fronthaul suffers from an inability to scale well with increased data rates and number of users [7], and inherently requires a Digital-to-Analog Converter (DAC) at each Remote Radio Head (RRH). These scalability issues can be alleviated through the use of Analog RoF (A-RoF). Although A-RoF would be a departure from currently deployed technologies, it harnesses the spectral efficiency of Multi-Carrier Modulation (MCM) wireless formats by transmitting them in their native analog form, while avoiding digitization, and thereby reducing hardware requirements.

Our previous work has focussed on the influence of the optical source(s) used in optical heterodyne systems using an A-RoF approach [8]. With specific regard to the low subcarrier baud rates associated with wireless MCM formats, we have previously reported that the mm-wave phase noise generated through optical heterodyning is the limiting factor on system performance. This leads to the requirement for the use of prohibitively low linewidth independent lasers, or pairs of coherent optical carriers, for sufficient performance. Coherent carriers may be generated using an Optical Frequency Comb (OFC), but even still, strict effective path matching between these carriers must be ensured throughout the system [9].

In this work, we propose a mm-wave optical heterodyne A-RoF system architecture which is robust both to phase noise generated through optical heterodyning, and carrier frequency offset arising from the frequency drift between two freerunning independent lasers. These features are enabled through the use of an analog mm-wave receiver which uses a phase noise correlated beat tone derived from the optical transmitter in lieu of a standalone Local Oscillator (LO). This 'LO-free' receiver implementation, which needs no additional phase noise or frequency offset estimation/correction, significantly reduces system complexity - adding to A-RoF's suitability for Ultra Dense (UD) deployment, and facilitating ease of transmitter-side photonic integration.

The work differs from previously demonstrated analog schemes in that it ultilizes mm-wave terms produced only by the interaction of the transmitted optical carriers in order to perform Phase Noise Cancellation (PNC). Similar implementations, [10] and [11], make use of electronic mixing and subtraction, respectively, but require a standalone optical LO at the receiver. [12] shows relaxed linewidth requirements and also avoids the use of a receiver LO, but introduces additional optical complexity in that separation of the terms to undergo PNC takes place in the optical domain.

\section{Experimental Setup and PNC Receiver}

\subsection{Optical heterodyne Testbed}

The experimental setup is shown in Fig. 1 with illustrative spectra along the system path. Two free-running commercial Tunable Lasers (TLs), each with a linewidth of $100 \mathrm{kHz}$ and output power of $+5 \mathrm{dBm}$, are operated at 193.394 and 193.45 $\mathrm{THz}$ respectively, giving a carrier frequency spacing, $\Delta F_{C}$, of $56 \mathrm{GHz}$. A Wavelength Selective Switch (WSS) is used to adjust the relative carrier levels to account for additional losses in the modulator arm, and to facilitate the generation of relatively strong carrier $\times$ carrier and carrier $\times$ signal $\mathrm{mm}-$ wave terms after heterodyning. After the two carrier are demultiplexed, Single-Sideband (SSB) modulation of one of the carriers is performed using an electrical $90^{\circ}$ hybrid and I/Q Mach Zehnder Modulator (I/Q MZM), while the other carrier is unmodulated. Optical Band-Pass Filters (OBPFs) are used 


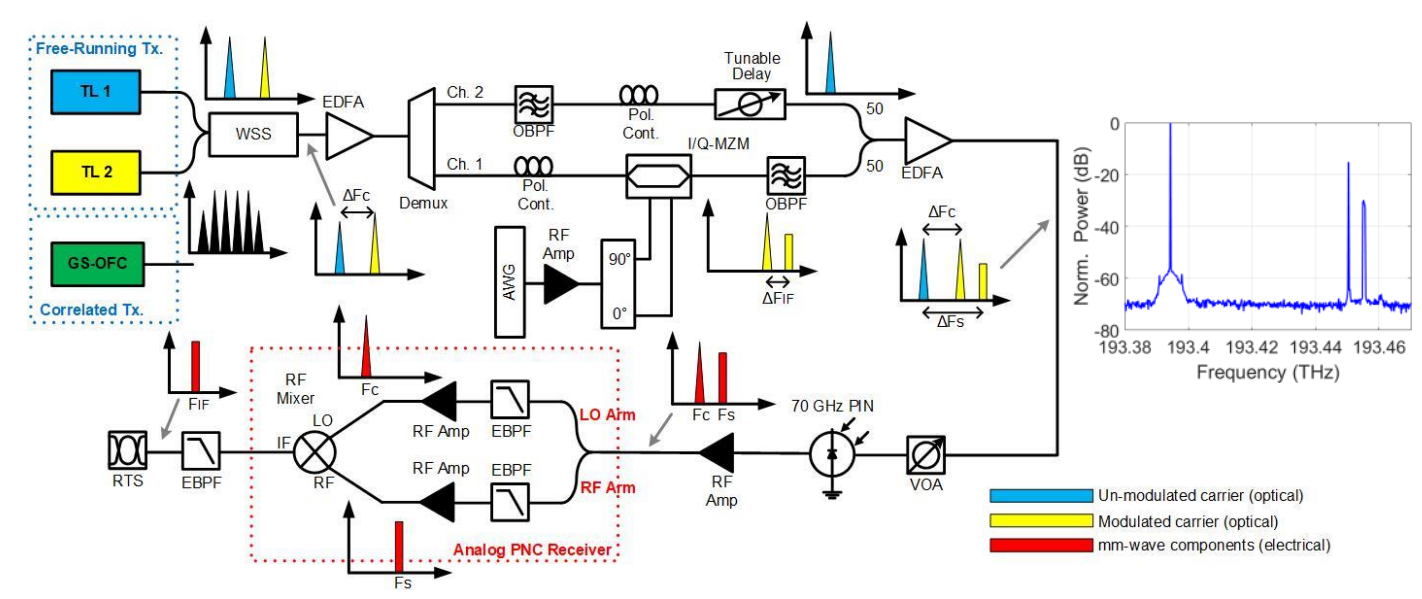

Fig. 1: Optical heterodyne mm-wave A-RoF experimental setup including figurative optical (blue and yellow) and electrical (red) spectra along the system path. The Phase Noise Cancelling analog receiver is outlined in red. The inset shows the measured optical spectrum at the output of the transmitter.

to suppress Amplified Spontaneous Emission (ASE) from the transmitter Erbium Doped Fiber Amplifier (EDFA).

Universally Filtered Orthogonal Frequency Division Multiplexing (UF-OFDM) - considered a candidate for future $5 \mathrm{G}$ communications [12] - is used as the analog MCM format. A single UF-OFDM band containing 76 subcarriers each modulated with 64 level Quadrature Amplitude Modulation (64-QAM) at 1.95 Mbaud - giving a total bandwidth of 148 $\mathrm{MHz}$ - is generated and digitally mixed to an intermediate frequency (IF) of $5 \mathrm{GHz}$. The signal is output from a Tektronix Arbitrary Waveform Generator (AWG) at $20 \mathrm{GSa} / \mathrm{s}$ and electrically amplified to $\sim 4 \mathrm{~V}_{\mathrm{pk}-\mathrm{pk}}$ before being used for optical SSB modulation.

The modulated and un-modulated optical carriers are combined and amplified by a transmitter booster EDFA before being sent through a short span of Standard Single Mode Fiber (SSMF) to the receiver. A Variable Optical Attenuator (VOA) is used to set the power falling on the $70 \mathrm{GHz}$ PIN photodetector (PD) to $+2 \mathrm{dBm}$. Photo-mixing of the optical frequency components at this stage produces electronic copies at beat frequencies arising from carrier $\times$ carrier mixing $\left(F_{C}=\right.$ $56 \mathrm{GHz})$ and un-modulated carrier $\times$ data sideband mixing $\left(F_{S}\right.$ $=61 \mathrm{GHz}$ ). These mm-wave terms are electrically amplified and sent to the PNC receiver. Wireless transmission is not carried out in this experimental work.

The signal is captured at the IF stage by a Tektronix Real Time Oscilloscope (RTS) sampling at $50 \mathrm{GSa} / \mathrm{s}$. Demodulation, Error Vector Magnitude (EVM) and Bit Error Rate (BER) calculations are completed offline. For comparison, results are also taken when (i) correlated optical carriers, derived from a Gain-Switched OFC (GS-OFC), are used for heterodyning, and (ii) when the 'LO arm' of the PNC receiver is replaced by an external standalone LO.

\subsection{Phase Noise Cancelling Receiver}

Phase noise cancellation and robustness to carrier frequency offset is achieved through the implementation of an analog 60 $\mathrm{GHz}$ receiver which is outlined by red dotted lines in Fig. 1. If the modulated optical carrier (yellow carrier in Fig. 1) remains un-suppressed after the modulation process, there will be a relatively strong beating term between this carrier and the unmodulated carrier (blue) - producing a mm-wave carrier at $F c$ after the PD. The receiver exploits the fact that this mm-wave carrier term at $F c$ will have the same phase noise as the mm-wave data term at $F_{S}$ (red spectra in Fig. 1); arising from the combined phase noise (linewidths) of the two optical sources. Isolating these $\mathrm{mm}$-wave frequency components, using an mm-wave splitter and Electrical BandPass Filtering (EBPF), allows the mm-wave carrier term at $F_{C}$ to be used as a phase noise correlated ' $L O$ '. This enables PNC and electronic down-conversion, in the same operation, as the two components are mixed to produce an IF data signal at the original $F_{I F}(5 \mathrm{GHz})$. For the receiver EBPFs shown in Fig. 1, the center frequencies and $3 \mathrm{~dB}$ bandwidths were: $f=56 \mathrm{GHz}$, $B W=500 \mathrm{MHz}$ (LO arm) and $f=62 \mathrm{GHz}, B W=6 \mathrm{GHz}(\mathrm{RF}$ data arm), respectively. The cancellation of phase noise in this manner is significant as it effectively removes the requirement for (and cost associated with) ultra-low linewidth optical sources which have been shown to be a necessity for heterodyne A-RoF applications [8].

Furthermore, if the laser sources, TL1 and TL2, are assumed to be independent, free-running, devices (i.e. uncorrelated) it is expected that their relative frequency drift over time will result in a time varying frequency separation between the un-modulated (blue in Fig. 1) and modulated (yellow) carriers (i.e. $\Delta F_{C}$ will vary over time). It follows that the frequency separation, $\Delta F_{S}$, between the un-modulated carrier (blue) and the data sideband (yellow) will also vary in exactly the same manner. Evidently, this will result in an equal shift, say $\delta F$, in the frequency of the mm-wave terms, $F_{C}$ and $F_{S}$, produced after the heterodyne operation at the PD. Critically, the difference between these terms will always equal the original IF, $F_{I F}$, regardless of the relative drift of the TLs i.e. if

$\boldsymbol{\Delta F}_{\boldsymbol{I F}}=\boldsymbol{\Delta} \boldsymbol{F}_{\boldsymbol{S}}-\boldsymbol{\Delta} \boldsymbol{F}_{\boldsymbol{C}} \quad$ [Optical domain @ TX.]

$\rightarrow \boldsymbol{F}_{\boldsymbol{I F}}=\boldsymbol{F}_{\boldsymbol{S}}-\boldsymbol{F}_{\boldsymbol{C}} \quad$ [Electronic domain @Rx.]

$\rightarrow \boldsymbol{F}_{I \boldsymbol{F}}=\left(\boldsymbol{F}_{S}+\boldsymbol{\delta} \boldsymbol{F}\right)-\left(\boldsymbol{F}_{\boldsymbol{C}}+\boldsymbol{\delta} \boldsymbol{F}\right)$

Achieving robustness to $\mathrm{CFO}$ in this way negates the requirement for frequency correlation, or locking, between the optical sources - significantly reducing transmitter complexity.

Since a phase and frequency correlated beat term (generated through the heterodyne process) is effectively used as the LO input to the IF mixer, a standalone LO is not required at the receiver. Adding to this, the fact that phase/frequency offset estimation/correction are negated, it is clear that the 
architecture presented permits a significant reduction in the complexity of the mm-wave transmitter and receiver designs. It should be noted that these advantages come at the cost of a requirement for the beat term to be transmitted wirelessly (after the PD) and in tandem with the data signal.

\section{Results and Discussion}

Using the free-running TLs, system performance is measured when the PNC is used and also where the 'LO arm' of the receiver is replaced by a standalone receiver LO operating at $56 \mathrm{GHz}$. Fig. 2 (a) shows perfect overlap between a portion of the transmitted (red) and received (blue) IF electrical spectra, and indicates that IF down-conversion through the PNC receiver makes the system agnostic to frequency fluctuations between the two TLs. After heterodyning, the mm-wave carrier phase noise is determined by a contribution of $100 \mathrm{kHz}$ (linewidth) from each TL. This indicates that insufficient RF carrier purity will be produced in order to support 64-QAM subcarrier modulation at 1.95 Mbaud [8]. However, by utilizing the proposed receiver architecture, $\mathrm{mm}$-wave $\mathrm{PNC}$ is enabled and a UF-OFDM signal with negligible phase noise can be detected as clearly shown by the received 64-QAM constellation (all subcarriers) in Fig. 2 (b). In this case the received EVM was $5.8 \%$ with BER measured to be $7.4 \times 10^{-5}$.

The equivalent spectra and constellation are shown in Fig. 2 (c) and (d) where a $56 \mathrm{GHz} \mathrm{LO}$ is used at the receiver. A carrier frequency offset of $\sim 4.5 \mathrm{MHz}$ can be observed in (c) due to the relative frequency drift of the TLs - which was observed over several captures to be a maximum of $10 \mathrm{MHz}$. In this case, the UF-OFDM signal could not be demodulated as indicated by the received constellation in (d). These results highlight the proposed system's robustness to both phase noise and carrier frequency offset arising from the use of unlocked and un-correlated lasers in a heterodyne configuration.

System performance is also measured, with and without the PNC receiver, in the case where two correlated optical carriers generated from a GS-OFC are isolated by the WSS and used for heterodyne detection. In this case, the tunable delay line shown in the un-modulated arm of the transmitter in Fig. 1 is used to vary the level of coherence between the two carriers [8]. Fig. 3 shows the received EVM as a function of optical delay between the tones; 0 to $300 \mathrm{ps}$. The comb lines lose coherence quickly due their high linewidth ( 80 $\mathrm{MHz}$ )/short coherence length [8] and this is reflected by the red data points which give performance in the case where an external LO is used at the receiver. Performance degrades from $6 \%$ to $13 \%$ EVM over the range as the comb lines become uncorrelated, and indicates the strict requirement for effective path length matching between the two carriers in this case. These requirements are relaxed when the standard receiver is replaced by the PNC receiver (green data points). Overall system performance is improved to below (or on) the 64-QAM FEC limit, with EVM ranging from 6\%-8.3\%. Perfect phase noise cancellation is not achieved at each delay value as the path lengths of the two arms in the PNC receiver are not exactly matched. This is due to the differences in the design of the two mm-wave EBPFs used coupled with the very large linewidths of the comb lines $(80 \mathrm{MHz})$. This issue could be easily overcome with an integrated PNC receiver where mmwave path matching would result in a performance of $6 \%$ over

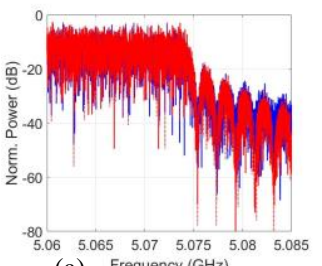
(a) Frequency $(\mathrm{GHz})$

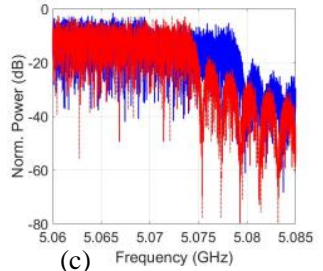

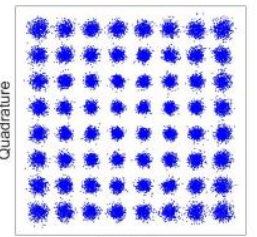

(b)

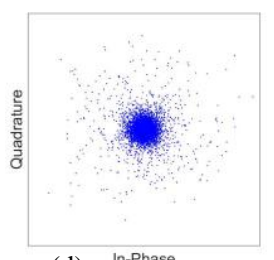

(d) In-Phase
Fig. 2: (a) and (b) shows a portion of the transmitted (red) and received (blue) IF UF-OFDM spectra, and the total received constellation, respectively, where the PNC receiver is used, (c) and (d) show the equivalent spectra and constellation where an external LO is used

the range. Finally, for comparative purposes, performances, where the two free-running TLs are used with the PNC receiver, are plotted (cyan data points). As expected, tuning the relative delay will not impact performance as the sources are inherently un-correlated. However, the result shows consistent performance of $\sim 6 \%$ EVM - once again highlighting the robustness of the proposed system architecture.

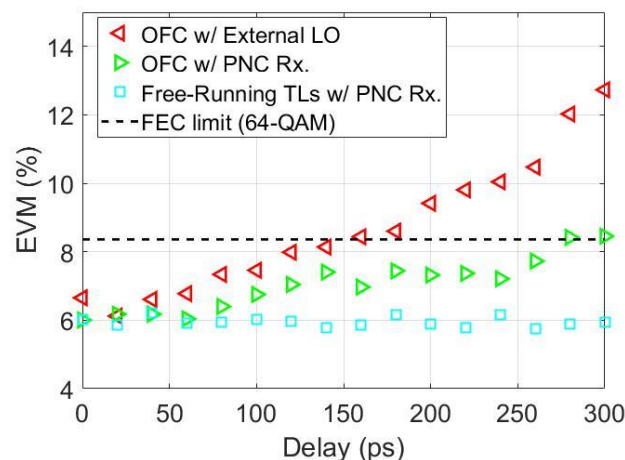

Fig. 3: EVM versus relative delay between the transmitted optical carriers with and without the PNC receiver.

\section{Conclusion}

In order to enable the type of high speed connectivity and applications envisaged through the use of mm-wave platforms, new technologies which reduce system complexity, facilitate dense RRH deployment, and which can be easily developed through photonic integration, are required. The system architecture presented in this work represents a significant reduction in the complexity of heterodyne based A-RoF mmwave systems. This is achieved, not only though avoidance of phase/frequency offset compensation digital or feedback circuitry at the receiver, but also by facilitating the use of uncorrelated lasers, with reduced source linewidth requirements - which has previously been shown to be a limiting factor for A-RoF based implementations. Results clearly indicate the proposed system's robustness to both phase noise and frequency offset when two free-running lasers are used for heterodyne operation, and a satisfactory performance of $\sim 6 \%$ EVM for 64-QAM UF-OFDM reception is achieved.

\section{Acknowledgement}

This work has emanated from research supported in part by a research grant from Science Foundation Ireland (SFI) under grant number 18/SIRG/5579 and is co-funded under the European Regional Development Fund under grant numbers 13/RC/2077, 12/RC/2276 and 15/US-C2C/I3132. 


\section{References}

[1] D. Choudhury, "5G wireless and millimeter wave technology evolution: An overview," proc. IEEE MTT-S International Microwave Symposium, Phoenix, USA, 2015, pp. 1-4.

[2] A. Kanno at al., "Field trial of 1.5-Gbps 97-GHz train communication system based on linear cell radio over fiber network for 240-km/h high-speed train," Proc. OFC Postdeadline Papers, San Diego, USA, March 2019, Th4C.2,

[3] M. Steeg et al., "Public field trial of a multi-RAT (60 GHz 5G/LTE/WiFi) mobile network," in IEEE Wireless Communications, 2018, 25 (5), pp. 38-46,

[4] D. Schulz et al., "Long-term outdoor measurements using a rate-adaptive hybrid optical wireless/60 GHz link over 100m," proc. ICTON 2017, Girona, Spain, pp. 1-4.

[5] S.E. Alavi et al., "Towards 5G: A photonic based millimeter wave signal generation for applying in $5 \mathrm{G}$ access fronthaul", Scientific Reports, 2016, 6 (19891)

[6] A. Stöhr, J. E. Mitchell and Y. Leiba, “Transparent wireless access to optical WDM networks using a novel coherent radioover-fiber (CRoF) approach," Proc. ICTON 2014, Graz, Austria, pp. 1-2.

[7] Thomas Pfeiffer, "Next Generation Mobile Fronthaul and Midhaul Architectures [Invited]," Journal of Optical Communications \& Networks, 2015, 7, B38-B45

[8] C. Browning et al., "Gain-Switched Optical Frequency Combs for Future Mobile Radio-Over-Fiber Millimeter-Wave Systems," in Journal of Lightwave Technology, 2018, 36 (19), pp. 4602-4610

[9] C. Browning, E. P. Martin, A. Farhang and L. P. Barry, "60 $\mathrm{GHz} 5 \mathrm{G}$ radio-over-fiber using UF-OFDM with optical heterodyning," in IEEE Photonics Technology Letters, 29 (23), pp. 2059-2062

[10] T. Kuri and K. Kitayama, "Optical heterodyne technique for densely multiplexed millimeter-wave-band radio-overfiber systems", IEEE Journal of Lightwave Technology, 2003, 21 (12), pp. 3167-3179

[11] J.P. Bekooij, "Method and device for compensating, in a coherent optical communication system with heterodyne detection, at the receiving side, phase noise of a transmitting laser and a local oscillator", US patent number 4918747, 17Apr, 1990.

[12] J. Li et al., "Millimeter-wave radio-over-fiber system based on two-step heterodyne technique", Optics Letters, 2009, 34 (20), pp. 3136-3138

[13] B. Farhang-Boroujeny and H. Moradi, “OFDM Inspired Waveforms for 5G," IEEE Communications Surveys \& Tutorials, 2016, 18 (4), pp. 2474-2492 